CERN-TH/98-74

LU TP 98-8

hep-ph/9804202

March 1998

\title{
Soft-Particle Spectra as a Probe of Interconnection Effects in Hadronic $\mathbf{W}^{+} \mathbf{W}^{-}$Events
}

\author{
Valery A. Khoze ${ }^{1}$ \\ INFN - Laboratori Nazionali di Frascati, \\ P.O. Box 13, I-00044 Frascati (Roma), Italy \\ and \\ TH Division, CERN, \\ CH-1211 Geneva 23, Switzerland \\ and \\ Torbjörn Sjöstrand ${ }^{2}$ \\ Department of Theoretical Physics, \\ Lund University, Lund, Sweden
}

\begin{abstract}
Cross-talk between the $\mathrm{W}^{+}$and $\mathrm{W}^{-}$sources of hadron production at LEP2 offers a hope to learn about basic properties of QCD, but at the same time threatens high-precision measurements of the $\mathrm{W}$ boson mass. Directly visible effects are not expected to be large, however. It is, therefore, important to develop methods to measure the level of interconnection in the data 'connectometers'. In this article we study one particular set of observables, namely the reduced rate of low-momentum particle production caused by reconnection. In realistic scenarios the expected signals are at the border of visibility, but not quite beyond reach. Special studies on kaons and protons, or with other subsets of the data, could provide supporting evidence. We also show how LEP1 $\mathrm{Z}^{0}$ events could be used to define an alternative reference sample.
\end{abstract}

\footnotetext{
${ }^{1}$ khoze@vxcern.cern.ch

${ }^{2}$ torbjorn@thep.lu.se
} 


\section{Introduction}

The accurate determination of the W-boson mass is one of the main objectives of LEP2. A statistical uncertainty on $M_{\mathrm{W}}$ of about half a per mille could eventually be achieved by reconstructing the $\mathrm{W}$ mass in hadronic WW decays. However, the systematic uncertainties due to hadronic final-state interactions and QCD interferences between the W decay products may induce substantial ambiguities, for reviews see $[1,2,3,4]$. Such an interconnection (cross-talk) between the $\mathrm{W}^{+}$and $\mathrm{W}^{-}$could occur because the two $\mathrm{W}$ bosons decay at short distances of order $1 / \Gamma_{\mathrm{W}} \sim 0.1 \mathrm{fm}$, and their decay products hadronize close to each other in space and time at the typical hadronic scale of $\sim 1 \mathrm{fm}$. The term 'interconnection' is a generic name covering those aspects of the final-state particle production that are not dictated by the separate decays of the $\mathrm{W}$ bosons, but can only be understood as a result of the joint action of the two.

The cross-talk between the $\mathrm{W}^{ \pm}$decay products undermines the traditional meaning of a $\mathrm{W}$ mass in the process

$$
\mathrm{e}^{+} \mathrm{e}^{-} \rightarrow \mathrm{W}^{+} \mathrm{W}^{-} \rightarrow \mathrm{q}_{1} \overline{\mathrm{q}}_{2} \mathrm{q}_{3} \overline{\mathrm{q}}_{4}
$$

called the $(4 \mathrm{q})$ mode. It is not even in principle possible to subdivide the final-state hadrons into two groups, one of which corresponds to the $\mathrm{W}^{+} \rightarrow \mathrm{q}_{1} \overline{\mathrm{q}}_{2}$ decay and the other to the $\mathrm{W}^{-} \rightarrow \mathrm{q}_{3} \overline{\mathrm{q}}_{4}$ decay: the identities of individual $\mathrm{W}^{ \pm}$decay products are not well-defined any more.

The strong-interaction dynamics induces a variety of interconnection effects between the hadronic decays of different W's, such as:

1. Quantum short-distance effects due to exchanges of perturbative gluons between the two initial q $\bar{q}$ systems.

2. Final-state radiative gluon interferences on the stage of parton-shower development.

3. Long-distance effects in the parton-to-hadron transition phase caused by a large overlap between the products of the two decays (non-perturbative rearrangement/reconnection).

4. Bose-Einstein (BE) correlations between identical bosons (in practice, pions).

As far as we are aware, the possibility of colour rearrangement in the process (1) was first considered in [5]. The rôle of QCD interconnection in hadronic WW decays in the framework of the $\mathrm{W}$ mass measurement was first discussed in [6]. This challenging topic has been quite intensively studied theoretically since then, see e.g. [7, 8, 9, 10, 11, 12]. $\mathrm{BE}$ correlations, in the W-mass context, were first discussed systematically in [13], with further studies since $[14,15,12]$. However, in this paper we do not attempt to cover the consequences of BE effects. The interest in QCD final-state interactions in the (4q) channel of $\mathrm{W}^{+} \mathrm{W}^{-}$decay has been strongly boosted by the prediction of the so-called colour-full scenario of [11], that the shift in $M_{\mathrm{W}}$ could be as large as several hundred $\mathrm{MeV}$.

It is necessary to emphasize that there is no question of whether interconnection between the W's exists or not; it is certainly there even in the QED context. ${ }^{3}$ Another wellknown precedent is $\mathrm{J} / \psi$ production in $\mathrm{B}$ decay: the $\mathrm{c} \overline{\mathrm{C}} \rightarrow \mathrm{J} / \psi$ transition requires a crosstalk between the two original colour singlets, $\overline{\mathrm{c}}+\mathrm{s}$ and $\mathrm{c}+$ spectator. The real challenge is

\footnotetext{
${ }^{3}$ The final-state QED interconnection induces a sizeable mass shift, $\left(\delta M_{\mathrm{W}}\right)_{\mathrm{QED}} \sim O\left(\alpha_{\mathrm{em}} \pi \Gamma_{\mathrm{W}}\right) \sim$ $50 \mathrm{MeV}$, in $\mathrm{e}^{+} \mathrm{e}^{-} \rightarrow 4$ fermions in the threshold region [16, 17]. However, at energies above $170 \mathrm{GeV}$, $\left(\delta M_{\mathrm{W}}\right)_{\mathrm{QED}} \sim O\left(\alpha_{\mathrm{em}} \Gamma_{\mathrm{W}} / \pi\right)$, and cannot exceed a few $\mathrm{MeV}[17,18]$.
} 
to understand how large the ambiguities for various observables can be. Evidently, it is not only the $\mathrm{W}$ mass that can be affected by interconnection. Various event characteristics in hadronic WW decay (such as the charged multiplicity or inclusive particle spectra) could, in principle, show effects even an order of magnitude bigger than that in $M_{\mathrm{W}}$, see e.g. [6]. On the other hand, in the inclusive cross section for process (1), the effects of the QCD (and QED) cross-talk are negligible [19]:

$$
\frac{\Delta \sigma_{\mathrm{WW}}^{\text {intercon }}}{\sigma_{\mathrm{WW}}} \sim\left(\frac{C_{F} \alpha_{\mathrm{s}}\left(\Gamma_{\mathrm{W}}\right)}{\pi}\right)^{2} \frac{1}{N_{C}^{2}-1} \frac{\Gamma_{\mathrm{W}}}{M_{\mathrm{W}}},
$$

where $C_{F}=\left(N_{C}^{2}-1\right) / 2 N_{C}, N_{C}=3$ being the number of colours.

A precise measurement of the $\mathrm{e}^{+} \mathrm{e}^{-} \rightarrow \mathrm{W}^{+} \mathrm{W}^{-}$threshold cross section (see e.g.[1] for details) would provide an interconnection-free method for measuring $M_{\mathrm{W}}$. Unfortunately, the combined total luminosity accumulated at LEP2 at a center-of-mass energy $\sqrt{s}=$ $161 \mathrm{GeV}$ (approximately $40 \mathrm{pb}^{-1}[20]$ ) is not sufficient to reach an interesting level of precision. So the direct kinematic reconstruction of $M_{\mathrm{W}}$ from the $\mathrm{W}$ hadronic decays remains the only realistic method at current and future energies of LEP2.

The potential significance of the cross-talk phenomena for the $\mathrm{W}$ mass reconstruction at LEP2 obviously warrants a detailed understanding of the size of the corresponding ambiguities. Note also that QCD reconnection is of interest in its own right, since it may provide us with a prospective laboratory for probing hadronization dynamics in space and time.

The perturbative aspects of QCD interconnection are, in principle, well controllable. Since the corresponding W mass shift is expected to be well within the uncertainties of the hadronization models (and about on the same level as QED corrections) we only recall here an estimate of [6],

$$
\left(\delta M_{\mathrm{W}}\right)_{\mathrm{PT}} \sim\left(\frac{C_{F} \alpha_{\mathrm{s}}\left(\Gamma_{\mathrm{W}}\right)}{\pi}\right)^{2} \frac{1}{N_{C}^{2}-1} \Gamma_{\mathrm{W}},
$$

which is of order of a few MeV. The perturbatively calculated mass-shift (as well as other observables) is colour suppressed, by two powers of $N_{C}$, which is typical for the gluon-mediated interaction between the two colour-singlet objects.

In the non-perturbative stage, which is our main concern in this paper, the coloursuppression situation varies between scenarios. Here factors like $1 / N_{C}^{2}$ may present, as in the perturbative phase, but they are multiplied by model-dependent coefficients, which are functions of the space-time variables. These coefficients, in principle, could be anything, even much larger than unity. For instance, in the models based on Lund string hadronization [21] the string itself consists of a multitude of coloured confinement gluons. Thus, if one gluon does not have the right colour to interact, then another nearby will. In such scenarios the colour suppression factor could well be compensated.

Since the space-time separation between the $\mathrm{W}^{+}$and $\mathrm{W}^{-}$decay vertices is typically of order $1 / \Gamma_{\mathrm{W}}$, only rather soft gluons (real or virtual) with an energy $k \lesssim \Gamma_{\mathrm{W}}$ could feel the collective action of both the $\mathrm{q}_{1} \overline{\mathrm{q}}_{2}$ and $\mathrm{q}_{3} \overline{\mathrm{q}}_{4}$ antennae/dipole systems, and thus participate in the cross-talk. This explains the origin of the last factor in eq. (3). Non-perturbative reconnection, which is our main concern, can occur wherever the hadronization regions of the two $\mathrm{W}$ bosons overlap. As was first emphasized in [6], the space-time picture of the evolution of the final state plays an essential rôle in understanding the size of the 
interconnection effects at the hadronic level. At the moment, given a lack of deep understanding of non-perturbative QCD physics, the possible consequences of the hadronic cross-talk between the W's can only be studied within the existing model-dependent Monte Carlo schemes of hadronization. These have done a very good job in describing a vast amount of information on hadronic $\mathrm{Z}^{0}$ decays, so one may expect that (after appropriate modifications) they could provide a reasonable estimate for the magnitude of interconnection-induced effects.

The currently used algorithms for treating the non-perturbative cross-talk $[1,2,3]$ all assume a local interaction, i.e. the decay products of two W's are mainly affected in the regions of overlap. Reconnection-unrelated parameters are tuned to optimize the agreement with $\mathrm{Z}^{0}$ data. Some models (not studied in this paper) allow reconnection also among the partons of a single $\mathrm{Z}^{0}$, and then consistency requires reconnection to be included in the above-mentioned tuning stage. The models are based on different philosophies and include various assumptions, in particular, concerning the possible rôle of non-singlet hadronization. Today we are still very far from claiming that there is one 'best' recipe; rather each approach may manifest some particular aspects of the true physics. This does not mean that all models have to be put on an equal footing: some may be "more equal than others".

Some essential phenomenological aspects appear to be common for different interconnection models:

1. The cross-talk dampens comparatively slowly with center-of-mass energy, $\sqrt{s}$, over the range that can be tested by LEP2.

2. Interconnection effects tend to be strongly dependent on the event topology, and could induce azimuthal anisotropies in the particle flow distributions.

3. The low-momentum final particles $(p \lesssim 1 \mathrm{GeV})$ are the main mediators in the hadronic cross-talk, and they are most affected by it.

4. Not far from the WW threshold the invariant mass of an original non-reconnected $q \bar{q}$ system is larger than that for a reconnected one. Therefore, it is not surprising that most of the model predictions show that the mean particle multiplicity in the (4q) mode, $\left\langle N^{(4 \mathrm{q})}\right\rangle$, is lower than twice the mean multiplicity of a hadronically decaying $\mathrm{W}$ in the mixed hadronic-leptonic channel $((2 \mathrm{q})$ mode $),\left\langle N^{(2 \mathrm{q})}\right\rangle,{ }^{4}$

$$
\frac{\left\langle N^{(4 \mathrm{q})}\right\rangle}{2\left\langle N^{(2 \mathrm{q})}\right\rangle}<1 \text {. }
$$

With increasing $\sqrt{s}$, the multiplicity in the purely hadronic final state may start to rise. ${ }^{5}$ However, at least within the models based on colour-confinement strings discussed in [6], the inequality (4) remains valid in the whole range of LEP2 energies.

5. All the models on the market (except of [11]) predict rather small cross-talk effects [1, $2,3,4]$. Thus, a conservative upper limit on the $M_{\mathrm{W}}$ shift seems to be something like around $50 \mathrm{MeV}$. Changes in the standard global event characteristics are expected at the per cent level. In marked difference with all other approaches, the colourfull scenario of [11] allows much larger signals. Thus, the $\mathrm{W}$ mass and the relative

\footnotetext{
${ }^{4}$ Contrary to frequent statements in the literature, there is no logical need to expect BE effects to increase $\left\langle N^{(4 \mathrm{q})}\right\rangle$, thus potentially compensating the reconnection-based prediction (4) (so-called BE conspiracy), see [15].

${ }^{5}$ The latter effect is easy to understand considering an energy far above threshold. Here both W's are strongly boosted away from each other, and a reconnection between the two widely separated systems leads to an increase in system masses, i.e. just opposite to the threshold behaviour.
} 
multiplicity shifts are predicted to be around $400 \mathrm{MeV}$ and $10 \%$, respectively. Note that the approach of [11] is the only available model which includes a non-singlet hadronization component. The strong claims of [11] have made the whole subject of 'connectometry' attractive for experimentalists.

The word connectometry is introduced here to cover various ways to detect interconnection-induced effects by measuring characteristics of the WW final state. The first experimental results on connectometry in the $\mathrm{W}^{+} \mathrm{W}^{-}$events have already been reported (for a review see [22]), and new experimental information continues to pour out from LEP2, see e.g. [23]. At the current level of statistics, there is no evidence for interconnection effects from the standard distributions in hadronic WW events. This agrees with the mainstream of model predictions, which suggests rather small effects. However, it should be remembered that a WW statistics larger by an order of magnitude is still to come.

An important point to bear in mind is that the values - even the signs — of shifts in various observables can depend strongly on the hadronization scenario and on the choice of model parameters. Moreover, results may be strongly sensitive to the adopted experimental strategy (jet reconstruction method, event selection, etc.).

It would be extremely valuable to establish a model-independent correlation between the shift in $M_{\mathrm{W}}$ and measurable quantities in the final-state distributions. Unfortunately, so far studies do not suggest any convincing correlation of such a type. Moreover, it appears that the measurements of different observables in a real-life experiment may require different event selections. So one has to proceed within the framework of a certain QCD Monte Carlo model. This paper, as well as our previous ones [6], is based on the models suggested by the Lund colour-confinement strings. One of our main objectives here is to establish how the interconnection-induced signal could be seen through the eyes of such a simple inclusive observable as the low-momentum particle spectrum. We will also study a possible calibration procedure based on $\mathrm{Z}^{0}$ events.

\section{Soft-Particle Spectra as connectometers}

$M_{\mathrm{W}}$ is not the best quantity for dealing with the interconnection effects. Firstly, the W mass shift depends quite strongly on its definition (shift of the peak position of the mass distribution, or the average mass shift, or ...) as well as on the reconstruction strategy. It looks like quite a tough task to learn anything about the structure of the QCD vacuum from what is encoded in $\delta M_{\mathrm{W}}$ only. Secondly, the reconnection signature in $M_{\mathrm{W}}$ is not large - it is only that we are set to measure this mass with the highest achievable accuracy that could make $\delta M_{\mathrm{W}}$ accessible. As has been already mentioned, many event characteristics may show relative effects, at least, an order of magnitude bigger.

The advantage of dealing with the standard global event shape characteristics for connectometry purposes was understood already from the beginning $[5,6,7]$. It has also been found that an observable could become more sensitive to cross-talk if it is probed within some selected kinematical regions. For instance, such a quantity as the charged multiplicity may allow a better sensitivity to interconnection if measured in restricted ranges of thrust $T$ or in special rapidity intervals. However, there is always a price to pay, namely lower event statistics and a possible dependence of the signature on the jet reconstruction method and experimental cuts.

There are several reasons to believe that the soft-particle yield could provide a suitable candidate for the rôle of connectometer: 
1. It is quite a general consequence of the space-time picture of the $\mathrm{WW} \rightarrow 4 \mathrm{q}$ process that the low-momentum particles $(p \lesssim 1 \mathrm{GeV})$ experience most directly the crosstalk effects.

2. The inclusive soft-momentum spectra of particles $h$ in the isolated individual $\mathrm{W} \rightarrow$ $2 \mathrm{q}$ decay, $\mathrm{d} n_{\mathrm{W}}^{h} / \mathrm{d} p$, can be rather well described, both within the QCD Monte Carlo models and with analytical perturbative techniques [24].

3. The $Z^{0}$ data provide an excellent experimental reference point, thanks to LEP1. When the $\mathrm{Z}^{0}$ results are used for calibration, the actual model dependence of the low-momentum spectra proves to be rather weak. Due to colour coherence in QCD cascades, the difference in the evolution scales corresponding to the $\mathrm{Z}^{0}$ and the $\mathrm{W}$ could cause only small changes (on the per cent level) at low momenta, see [25] and references therein. Effects due to the difference in the primary quark flavour composition also remain on the per cent level for soft particles (see e.g. [26] for a comparison between the $\mathrm{Z}^{0} \rightarrow \mathrm{b} \bar{b}$ and $\mathrm{Z}^{0} \rightarrow \mathrm{q} \overline{\mathrm{q}}$ events). Such small corrections could readily be accounted for.

4. Inclusive soft-particle spectra are fairly independent of the jet reconstruction strategy and event selection.

To implement colour reconnection we follow below the ideas and techniques that were developed in [6]. Two main scenarios are called 'Type I' and 'Type II' in analogy with the two types of superconducting vortices which could correspond to colour strings. In the 'Type I' models the reconnection probability depends on the integrated space-time overlap of the extended strings (elongated bags) formed in the two $\mathrm{W}$ decays. In the 'Type II', the reconnection occurs at the crossing of two string cores (vortex lines). The 'Type $\mathrm{II}^{\prime}$ ' is a variant of the latter, with the requirement that a reconnection is allowed only if it leads to a reduction of the string length. We also present results corresponding to a simplified implementation (by us, using JETSET [27] rather than ARIADNE [28] showers) of the ' $\mathrm{GH}$ ' model [7], where the reconnection is selected solely based on the criterion of a reduced string length. Numbers are also shown for two toy models, the 'instantaneous' and 'intermediate' scenarios of [6]. In the former (which is equivalent to that in [5]) the two reconnected systems $\mathrm{q}_{1} \overline{\mathrm{q}}_{4}$ and $\mathrm{q}_{3} \overline{\mathrm{q}}_{2}$ are immediately formed and then subsequently shower and fragment independently of each other. In the latter, a reconnection occurs between the shower and fragmentation stages. One has to bear in mind that the last two 'optimistic' (from the connectometry point of view) toy approaches are oversimplified extremes and are not supposed to correspond to the true nature. These scenarios may be useful for reference purposes, but their experimental rejection can at most be considered as warmup exercises for the real task.

Within these models there are some general qualitative predictions for the soft-particle spectra in the $\mathrm{WW} \rightarrow 4 \mathrm{q}$ events, $\mathrm{d} n_{4 \mathrm{q}}^{h} / \mathrm{d} p$, in the LEP2 energy range.

1. Depopulation of the low-momentum hadrons, relative to the no-reconnection scenario, due to the Lorentz boosts of the alternative $q_{1} \bar{q}_{4}$ and $q_{3} \bar{q}_{2}$ dipoles/antennae.

2. As in the case of the well-known standard string effect [21, 29], such a depopulation should become more pronounced for heavier hadrons $(K, p, \ldots)$. An unseparated sample of kaons and protons would also be less affected by the BE phenomenon.

3. A gradual reduction of the cross-talk with center-of-mass energy, since the two outgoing $\mathrm{W}$ hadronic systems are more and more boosted apart.

It is worthwhile to emphasize that a final-state observable may well have different sensitivity levels to different interconnection effects. Thus, connectometry based on the soft- 
particle yield is supposed to serve mostly as a hearing-aid for the long-distance hadronic cross-talk. The readers are reminded that the very idea of such studies has emerged from the string/dipole fragmentation picture. By contrast, it cannot be ruled out that $\mathrm{BE}$ effects will not be seen in inclusive spectra, and only emerge in particle correlations. Furthermore, low-momentum spectra do not look like a proper diagnostic tool to probe virtual gluon (photon) interference phenomena. As an instructive example, consider QED Coulomb effects on the $M_{\mathrm{W}}$ reconstruction $[16,17]$. While the $\mathrm{W}$ line shape is sizeably affected in the threshold region (at the $50 \mathrm{MeV}$ level), it is quite unlikely one would get any significant distortions in the inclusive final hadron spectra.

\section{$3 \quad$ Numerical Results}

Armed with the algorithms of $[6,7]$ we can now proceed to calculate the effects of hadronic cross-talk on the low-momentum distributions. Note that the momentum spectra in the following are considered as totally inclusive quantities. So all hadronic WW events are included, without any cuts. It is up to experimentalists to select cuts which reduce the background and to correct for that.

Results have been obtained with PyтHIA version 6.1 [27]. All numbers are based on samples of 400,000 events per model. The quoted number of decimals at times is larger than warranted by statistical accuracy, in order to simplify comparisons. Systematic errors, of course, by far exceed statistical ones, but generally divide out in comparisons. (For instance, those coming from different tunes of fragmentation parameters to the LEP1 data.)

To illustrate the size of the expected effects, consider first the energy $\sqrt{s}=172 \mathrm{GeV}$. The ratio of the momentum spectrum of charged particles with reconnection to that without it (a hypothetical no-reconnection case which is equivalent to the mixed leptonichadronic (2q) channel) is shown in Fig. 1 for various scenarios. The nominal input $M_{\mathrm{W}}$ is $80.33 \mathrm{GeV}$, and QED initial-state radiation (ISR) is not included. Since the main changes occur in the low-momentum range, we plot in Fig. 2 the same distributions as in Fig. 1 but only for momenta up to $2 \mathrm{GeV}$.

As expected, a suppression of the ratio at low momenta is quite visible. Note that the vertical scales are quite different in the (a) and (b) frames. We have to warn the reader that the ' $\mathrm{GH}$ ' model contains the reconnection probability as a free parameter, here set to unity. If one assumes reconnection at the $30 \%$ level, as is the order obtained in the II and $\mathrm{II}^{\prime}$ scenarios and is assumed in the I one, the 0.9 level of ' $\mathrm{GH}$ ' at very low values of $p$ would change to 0.97 , which is comparable with the above three models. It is thus only the unrealistic intermediate and instantaneous scenarios that deviate from the rest, by giving significantly larger suppressions.

The 'shape universality' among the realistic models could be related to all of them being based on the same string fragmentation framework, but we would like to believe that it may be more general than that. (Obviously a point well worth studying further.) If so, we here have a good gauge for the reconnection probability. However, remember that models with the same probability may well give different $\mathrm{W}$ mass shifts, so we are still not gauging the key quantity.

Note that the phase space for particle production behaves like $p^{2} \mathrm{~d} p$ at small $p$. The region $p<0.2 \mathrm{GeV}$ therefore contains few particles and is sensitive to statistical fluctuations. The large spikes seen in the first bin or two of Fig. 2 should therefore not be 

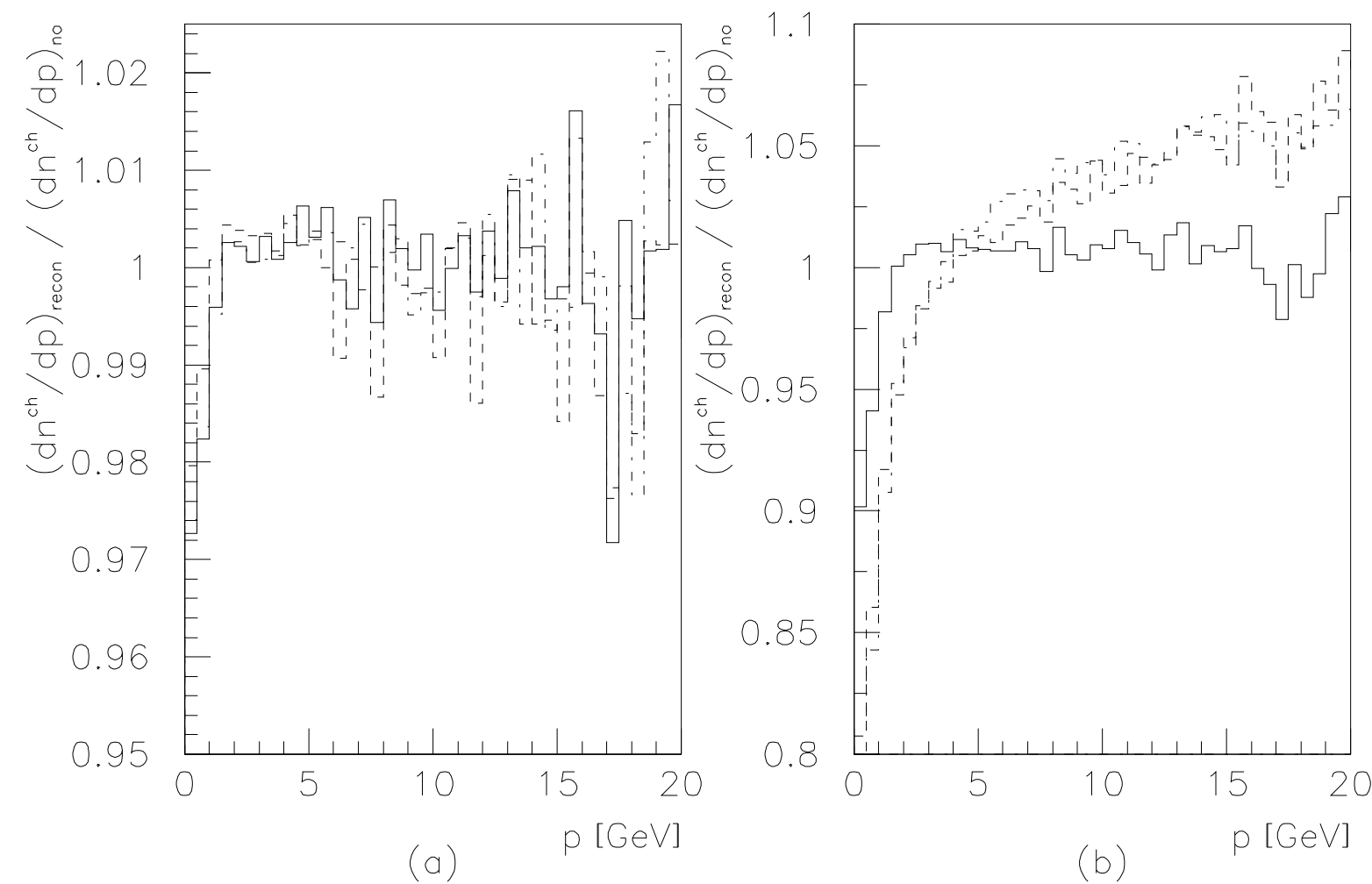

Figure 1: Ratio of reconnection to no-reconnection momentum spectra $\mathrm{d} n^{\mathrm{ch}} / \mathrm{d} p$ for all charged particles with $p<20 \mathrm{GeV}$. a) Model I full, II dashed and II' dashed-dotted. b) ' $\mathrm{GH}$ ' full, intermediate dashed and instantaneous dashed-dotted. Note difference in vertical scales. Energy is $172 \mathrm{GeV}$; no ISR.
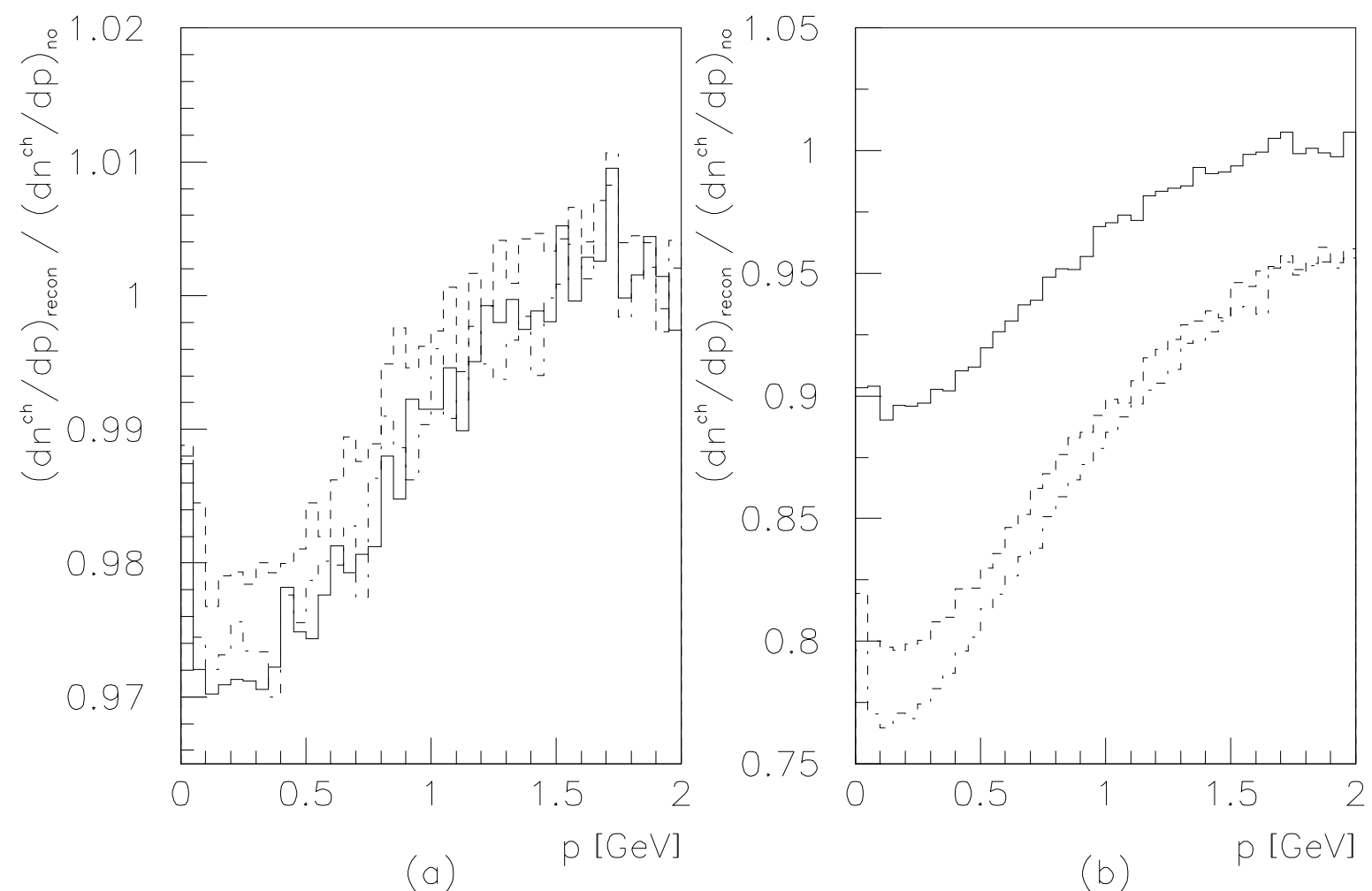

Figure 2: Ratio of charged momentum spectra as in Fig. 1, for the low-momentum region $p<2 \mathrm{GeV}$. 


\begin{tabular}{|l|c|c|c|c|}
\hline & \multicolumn{2}{|c|}{$\sqrt{s}=172 \mathrm{GeV}$} & \multicolumn{2}{c|}{$\sqrt{s}=195 \mathrm{GeV}$} \\
\hline model & $\left\langle N_{\mathrm{ch}}^{(4 \mathrm{q})}\right\rangle$ & $\left\langle N_{\mathrm{ch}}^{(4 \mathrm{q})}\right\rangle_{p<1 \mathrm{GeV}}$ & $\left\langle N_{\mathrm{ch}}^{(4 \mathrm{q})}\right\rangle$ & $\left\langle N_{\mathrm{ch}}^{(4 \mathrm{q})}\right\rangle_{p<1 \mathrm{GeV}}$ \\
\hline no-reconnection & 38.264 & 15.141 & 38.385 & 14.175 \\
\hline I & 37.941 & 14.802 & 38.230 & 14.025 \\
II & 38.070 & 14.909 & 38.284 & 14.063 \\
II' & 37.958 & 14.822 & 38.223 & 14.009 \\
\hline 'GH' & 37.108 & 13.955 & 37.685 & 13.502 \\
Intermediate & 35.259 & 12.631 & 35.597 & 12.227 \\
Instantaneous & 34.929 & 12.303 & 36.770 & 12.749 \\
\hline
\end{tabular}

Table 1: Charged multiplicity, in total and for $p<1 \mathrm{GeV}$, at 172 and $195 \mathrm{GeV}$, respectively. No ISR.

overinterpreted. In particular, the same no-reconnection event sample is used as denominator for all the ratios, so a downwards fluctuation here could be the common cause for all the spikes.

To further quantify the expectations we give in Tab. 1 the results for the mean charged multiplicity $\left\langle N_{\mathrm{ch}}^{(4 \mathrm{q})}\right\rangle$ together with the charged multiplicity in the momentum range $p<1 \mathrm{GeV},\left.\left\langle N_{\mathrm{ch}}^{(4 \mathrm{q})}\right\rangle\right|_{p<1 \mathrm{GeV}}$. The multiplicity difference between models is almost entirely coming from $p<1 \mathrm{GeV}$, except in the intermediate and instantaneous scenarios, where reconnection effects extend further out in momentum. Some increase of multiplicity outside of the centrally depleted region is a direct consequence of energy conservation, however.

Note that the averaged measured value of $\left\langle N_{\mathrm{ch}}^{(4 \mathrm{q})}\right\rangle$ at $\sqrt{s}=172 \mathrm{GeV}[22]$,

$$
\left\langle N_{\mathrm{ch}}^{(4 \mathrm{q})}\right\rangle_{\exp }=38.94 \pm 1.07
$$

is in a good agreement with the no-reconnection prediction.

To examine the rôle of the hadron mass we plot in Figs. 3 and 4 the ratio of the $\mathrm{K}^{ \pm}$plus $\mathrm{p}, \overline{\mathrm{p}}$ spectra for the same six models as before. This non-separated sample of heavy charged particles is experimentally more convenient to study than either of $\mathrm{K}$ or $\mathrm{p}$ separately, and carries the same physics message. As anticipated, the difference relative to the no-reconnection scenario becomes more marked in the heavy-charged-particle case. Moreover, the depletion now occurs in a somewhat wider momentum region. However, at the present stage we do not want to make too optimistic claims here. The chances of success of such studies may well be endangered by low statistics (recall that $\mathrm{K}+\mathrm{p}$ are less than $10 \%$ of the soft particles) and the detection efficiency. All such issues need a lot of further work. For instance, a promising experimental approach is now suggested [30], based on the combination of the $\mathrm{d} E / \mathrm{d} x$ and RICH techniques for tagging low-momentum heavy hadrons. In any case, one can cautiously say that if/when the reconnection signal is established safely in the charged-particle spectra, the $\mathrm{K}+\mathrm{p}$ sample would provide additional instructive information.

As has been mentioned already, we expect a gradual decrease of the cross-talk signal above threshold. In order to quantify this effect we present in Fig. 5 the ratio of momentum spectra in the range $p<2 \mathrm{GeV}$ at $\sqrt{s}=195 \mathrm{GeV}$. The numbers corresponding to this energy are shown in Tab. 1. The message from comparisons between the results at the two energies is quite clear: at $\sqrt{s}=195 \mathrm{GeV}$ the differences drop down to about a half 

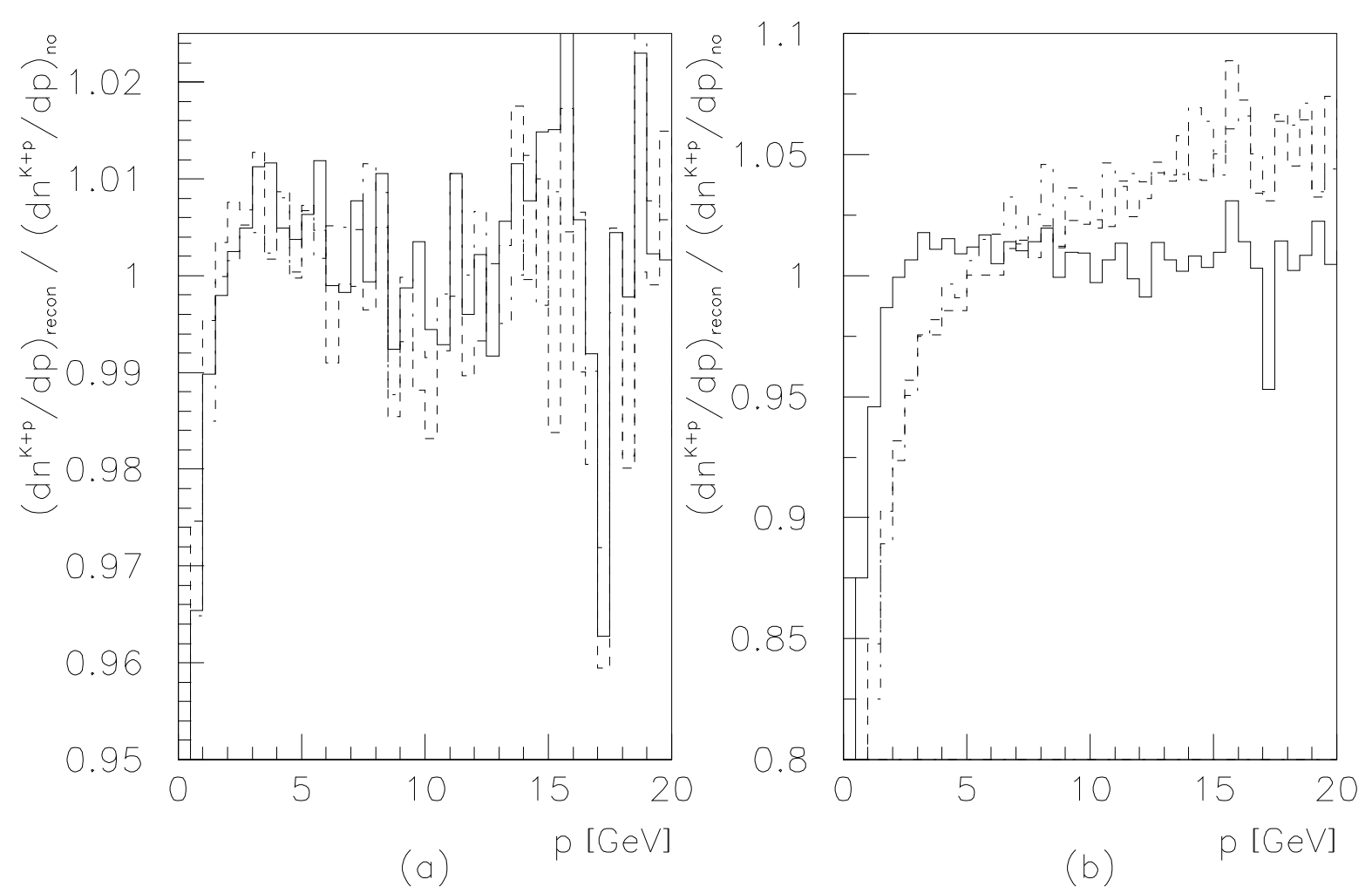

Figure 3: Ratio of reconnection to no-reconnection momentum spectra $\mathrm{d} n^{\mathrm{K}+\mathrm{p}} / \mathrm{d} p$ for heavy charged particles $\left(\mathrm{K}^{ \pm}\right.$and $\left.\mathrm{p}, \overline{\mathrm{p}}\right)$ only, with $p<20 \mathrm{GeV}$. Energy is $172 \mathrm{GeV}$; no ISR. Model curves as in Fig. 1.
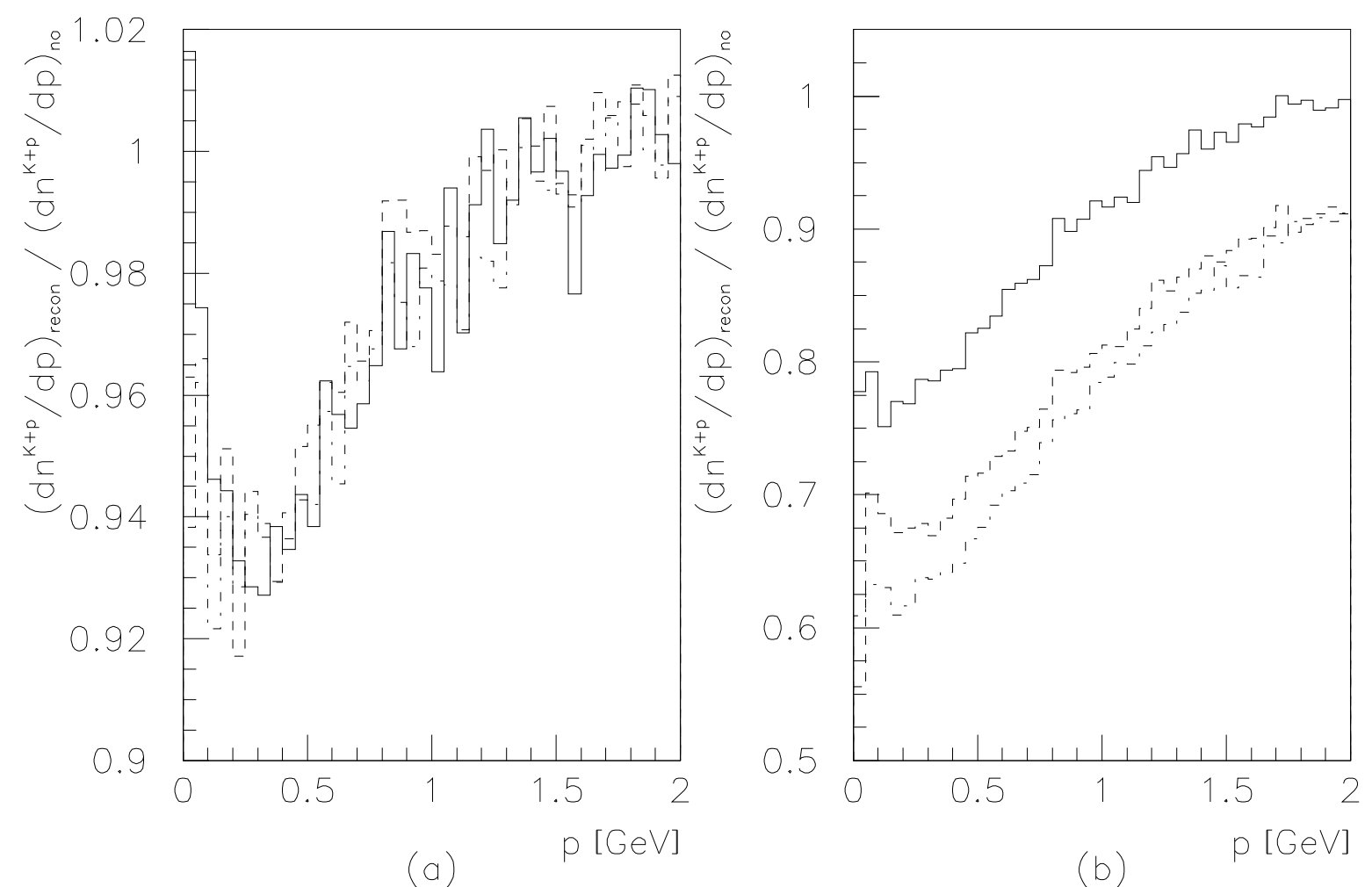

Figure 4: Ratio of heavy charged particle momentum spectra as in Fig. 3, for the lowmomentum region $p<2 \mathrm{GeV}$. 

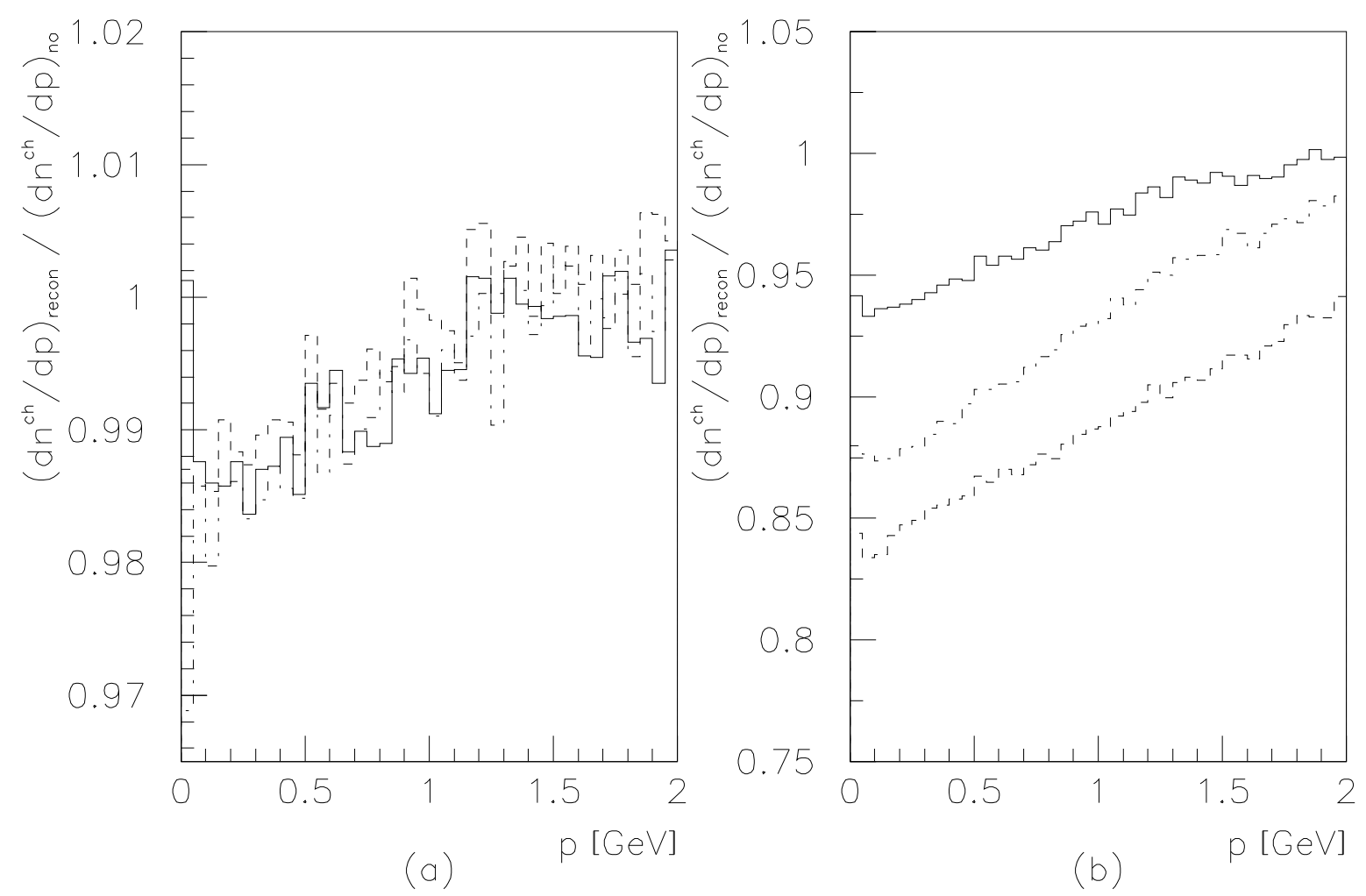

Figure 5: Ratio of charged momentum spectra as in Fig. 2, for the low-momentum region $p<2 \mathrm{GeV}$, but at an energy of $195 \mathrm{GeV}$. No ISR.

of what they were at $172 \mathrm{GeV}$. Recalling that the LEP2 statistics leans towards higher energies, this result naturally does not make the prospects of connectometry at LEP2 more optimistic.

For the $\mathrm{W}$ mass shift itself, the story is more complicated. At a higher energy the two W's are more boosted apart, so a fixed change of low-momentum particles could have a larger impact on the $\mathrm{W}$ mass. Over the LEP2 energy range the net effect, of a decreasing change of the momentum spectra with an increasing specific impact, is an essentially unchanged uncertainty in the $\mathrm{W}$ mass. That is, the shift in individual models may move in either direction as a function of energy, but for the 'envelope' of reasonable models there is no reason to expect neither a reduction nor an increase in the level of uncertainty.

We show below some results of further studies, in order to provide a more quantitative understanding of how large effects are to be expected under different requirements.

Tab. 2 shows the numbers at $\sqrt{s}=172 \mathrm{GeV}$, without inclusion of ISR effects. The first line represents the reference numbers without reconnection. In addition to the total multiplicity, we give the multiplicity in the range $0.15<p<0.7 \mathrm{GeV}$, which roughly maximizes the size of reconnection effects. (A smaller range would reduce statistics, a larger reduce the average level of effects.) The mass shift $\left\langle\delta M_{\mathrm{W}}\right\rangle$ is defined as

$$
\left\langle\delta M_{\mathrm{W}}\right\rangle=\left\langle M_{\mathrm{W}}\right\rangle_{\mathrm{recon}}-\left\langle M_{\mathrm{W}}\right\rangle_{\mathrm{no}-\text { recon }}
$$

For illustration purposes we have chosen mass reconstruction method 3 of [6]. The basic idea of this method is to minimize the sum of deviations from an $80 \mathrm{GeV}$ nominal mass, $\left|M_{\mathrm{W}}^{(1)}-80 \mathrm{GeV}\right|+\left|M_{\mathrm{W}}^{(2)}-80 \mathrm{GeV}\right|$, where $M_{\mathrm{W}}^{(1,2)}$ are the two reconstructed jet-jet masses. (The exact value used for the nominal mass is not critical; the idea is only to reject combinations that are clearly unreasonable.) The minimization is performed over the 


\begin{tabular}{|l|c|c|c|c|r|}
\hline & \multicolumn{4}{|c|}{ particle species and $p$ range $(\mathrm{GeV})$} & \\
\hline model & $\begin{array}{c}\text { charged } \\
\text { all }\end{array}$ & $\begin{array}{c}\text { charged } \\
0.15-0.7\end{array}$ & $\begin{array}{c}\mathrm{K}^{ \pm} \\
0.15-0.7\end{array}$ & $\begin{array}{c}\mathrm{p}, \overline{\mathrm{p}} \\
0.15-0.7\end{array}$ & $\begin{array}{r}\left\langle\delta M_{\mathrm{W}}\right\rangle \\
(\mathrm{MeV})\end{array}$ \\
\hline no-reconnection & 38.264 & 10.099 & 0.495 & 0.159 & - \\
\hline $\mathrm{I} /$ no-rec & 0.992 & 0.975 & 0.951 & 0.923 & 7 \\
$\mathrm{II} /$ no-rec & 0.995 & 0.982 & 0.958 & 0.928 & -5 \\
$\mathrm{II} /$ no-rec & 0.992 & 0.976 & 0.952 & 0.922 & -4 \\
\hline 'GH'/no-rec & 0.970 & 0.911 & 0.842 & 0.746 & -55 \\
intermediate/no-rec & 0.922 & 0.820 & 0.730 & 0.647 & 333 \\
instantaneous/no-rec & 0.913 & 0.797 & 0.697 & 0.582 & 1054 \\
\hline
\end{tabular}

Table 2: Results on $\left\langle N_{\mathrm{ch}}\right\rangle,\left\langle N_{\mathrm{K}}\right\rangle$ and $\left\langle N_{\mathrm{p}, \overline{\mathrm{p}}}\right\rangle$ in different momentum intervals. The reconnection numbers are normalized to the no-reconnection numbers in the first row. Also the reconnection-induced W mass shifts $\left\langle\delta M_{\mathrm{W}}\right\rangle$ are shown. Energy is $172 \mathrm{GeV}$; no ISR.

\begin{tabular}{|l|c|c|c|c|r|}
\hline & \multicolumn{4}{|c|}{ particle species and $p$ range $(\mathrm{GeV})$} & \\
\hline model & $\begin{array}{c}\text { charged } \\
\text { all }\end{array}$ & $\begin{array}{c}\text { charged } \\
0.15-0.7\end{array}$ & $\begin{array}{c}\mathrm{K}^{ \pm} \\
0.15-0.7\end{array}$ & $\begin{array}{c}\mathrm{p}, \overline{\mathrm{p}} \\
0.15-0.7\end{array}$ & $\begin{array}{r}\left\langle\delta M_{\mathrm{W}}\right\rangle \\
(\mathrm{MeV})\end{array}$ \\
\hline no-reconnection & 38.385 & 9.357 & 0.430 & 0.124 & - \\
\hline $\mathrm{I} /$ no-rec & 0.996 & 0.989 & 0.976 & 0.966 & 19 \\
$\mathrm{II} /$ no-rec & 0.997 & 0.991 & 0.980 & 0.966 & -28 \\
$\mathrm{II} /$ no-rec & 0.996 & 0.987 & 0.978 & 0.952 & -26 \\
\hline 'GH'/no-rec & 0.982 & 0.948 & 0.912 & 0.895 & 92 \\
Intermediate/no-rec & 0.927 & 0.858 & 0.825 & 0.838 & 307 \\
Instantaneous/no-rec & 0.958 & 0.892 & 0.840 & 0.811 & 1473 \\
\hline
\end{tabular}

Table 3: Results as in Tab. 2, but at $195 \mathrm{GeV}$. No ISR.

\begin{tabular}{|l|c|c|c|c|r|}
\hline & \multicolumn{4}{|c|}{ particle species and $p$ range $(\mathrm{GeV})$} & \\
\hline model & $\begin{array}{c}\text { charged } \\
\text { all }\end{array}$ & $\begin{array}{c}\text { charged } \\
0.15-0.7\end{array}$ & $\begin{array}{c}\mathrm{K}^{ \pm} \\
0.15-0.7\end{array}$ & $\begin{array}{c}\mathrm{p}, \overline{\mathrm{p}} \\
0.15-0.7\end{array}$ & $\begin{array}{r}\left\langle\delta M_{\mathrm{W}}\right\rangle \\
(\mathrm{MeV})\end{array}$ \\
\hline no-reconnection & 38.388 & 9.445 & 0.436 & 0.127 & - \\
\hline $\mathrm{I} /$ no-rec & 0.995 & 0.986 & 0.974 & 0.969 & 36 \\
$\mathrm{II} /$ no-rec & 0.996 & 0.987 & 0.977 & 0.953 & -19 \\
$\mathrm{II} /$ no-rec & 0.995 & 0.984 & 0.970 & 0.957 & -21 \\
\hline 'GH'/no-rec & 0.980 & 0.943 & 0.905 & 0.878 & 80 \\
Intermediate/no-rec & 0.926 & 0.851 & 0.817 & 0.824 & 291 \\
Instantaneous/no-rec & 0.953 & 0.880 & 0.825 & 0.779 & 1360 \\
\hline
\end{tabular}

Table 4: Results as in Tabs. 2 and 3, but at $195 \mathrm{GeV}$ and with ISR included.

three distinct ways to pair the four jets. Note that the numbers in the last column in Tab. 2 do not coincide with the numbers in [6]: in the current study all WW events are considered, while jet angular separation and minimum energy cuts were applied in [6].

Apart from the spread of $\left\langle\delta M_{\mathrm{W}}\right\rangle$ values among the four realistic models, also note that the $\left\langle\delta M_{\mathrm{W}}\right\rangle$ of the intermediate and instantaneous toy models differ by about a factor of 
three, ${ }^{6}$ although the depletion of low-momentum particles is fairly similar. This drives home the point that a depletion measurement does not necessarily provide a correction procedure for the $\mathrm{W}$ mass determination. At best, an observation of no depletion could help reduce the systematic uncertainty that should be assigned to the mass.

The message from comparison between the numbers in the 'charged', ' $\mathrm{K}^{ \pm}$' and ' $\mathrm{p}, \overline{\mathrm{p}}$ ' columns in Tab. 2 is that there is no special need for a complete $\pi / \mathrm{K} / \mathrm{p}$ separation - one can rest content with a more relaxed separation $\pi /$ non- $\pi$. (Primary produced electrons and muons are of negligible importance, so we do not here address how they should be classified experimentally.) We also remind that $\mathrm{K}_{\mathrm{S}}^{0}$ behaves the same way as $\mathrm{K}^{ \pm}$and that $\Lambda$ and other strange baryons very closely follow the same pattern as p. Therefore a rather inclusive tagging by secondary vertices (in the range above charm and bottom decays) fills a similar function as a non- $\pi$ sample of charged particles.

Since LEP2 statistics is biased towards higher energies, in Tab. 3 we give the numbers for $\sqrt{s}=195 \mathrm{GeV}$, still without ISR corrections. Comparing the ratios in Tab. 3 with those in Tab. 2 one observes once more the anticipated reduction of the signal with centerof-mass energy.

One may worry that the presence of ISR might smear out momentum distributions, and thus dilute the reconnection signal. The momentum range where the ratio of the spectra drops below unity might also extend outwards. However, given that the boosts induced by the ISR are of rather moderate magnitude, at least on the scale considered here, the effects are not expected to be large. To quantify the possible impact of ISR corrections, we give in Tab. 4 the results corresponding to the same parameters as in Tab. 3, but with ISR effects included. A comparison of the results in the two tables do not show any essential changes in particle number ratios. If anything, the ISR enhances the signals, by shifting the WW system to a lower invariant mass, thus counteracting the general drop of effects with increasing energy. We note that the impact of ISR is small also at $172 \mathrm{GeV}$ (not shown), as a trivial consequence of the reduced phase space for photon emission. So ISR corrections cannot make a significant impact on the conclusions of this paper. By contrast, the reconnection mass shift is affected by ISR, both at 172 and $195 \mathrm{GeV}$, thus reflecting the non-negligible dependence of $\left\langle\delta M_{\mathrm{W}}\right\rangle$ on the details of the (simulated) reconstruction procedures.

The main lesson from the analysis of fully inclusive spectra is not too encouraging: the effects predicted within the realistic models in a real-life experiment seems to be quite small. In order to enhance the signature for hadronic cross-talk one may wish to examine more specific measures, i.e. search for the signal in the regions of phase-space where the effects are expected to be most pronounced.

It has been understood from the very beginning $[5,6,7]$ that cross-talk should be enhanced in event configurations with large thrust $T$ (or, equivalently, small separation angle between initial cross-talking q $\bar{q}$ pairs). To quantify the possible impact of the largethrust selection on the low-momentum spectra we show in Tab. 5 the numbers for a trust cut of $0.8<T<0.9$ at $172 \mathrm{GeV}$, without ISR effects included. The region $T>0.9$ contains few $\mathrm{W}^{+} \mathrm{W}^{-}$events and a large background from ordinary $\mathrm{e}^{+} \mathrm{e}^{-} \rightarrow \gamma^{*} / \mathrm{Z}^{0 *} \rightarrow \mathrm{q} \overline{\mathrm{q}}$

\footnotetext{
${ }^{6}$ Naively, the instantaneous mass shift could have been even larger. However, most events do not have a large amount of wide-angle radiation, where the coherence pattern of the radiation would be of importance. Also, most of the time the four quarks are not moving in directions close to each other and, when they are, the charge- and parity-symmetry-breaking structure of the $\mathrm{W}$ decays preferentially puts two quarks in the same hemisphere rather than a $\mathrm{q}$ from one $\mathrm{W}$ and a $\overline{\mathrm{q}}$ from the other. Thus the reconnected systems are closer to the original ones than pure random.
} 


\begin{tabular}{|l|c|c|c|c|c|r|}
\hline & & \multicolumn{6}{|c|}{ particle species and $p$ range $(\mathrm{GeV})$} & \\
\hline model & $\begin{array}{c}\text { survival } \\
\text { rate }\end{array}$ & $\begin{array}{c}\text { charged } \\
\text { all }\end{array}$ & $\begin{array}{c}\text { charged } \\
0.15-0.7\end{array}$ & $\begin{array}{c}\mathrm{K}^{ \pm} \\
0.15-0.7\end{array}$ & $\begin{array}{c}\mathrm{p}, \overline{\mathrm{p}} \\
0.15-0.7\end{array}$ & $\begin{array}{r}\left\langle\delta M_{\mathrm{W}}\right\rangle \\
(\mathrm{MeV})\end{array}$ \\
\hline no-reconnection & 0.343 & 37.207 & 9.683 & 0.479 & 0.157 & - \\
\hline $\mathrm{I} /$ no-rec & 0.344 & 0.992 & 0.978 & 0.966 & 0.935 & -1 \\
$\mathrm{II} /$ no-rec & 0.342 & 0.996 & 0.984 & 0.963 & 0.947 & -6 \\
$\mathrm{II} /$ no-rec & 0.344 & 0.993 & 0.980 & 0.960 & 0.940 & -20 \\
\hline 'GH'/no-rec & 0.346 & 0.972 & 0.921 & 0.862 & 0.800 & -58 \\
Intermediate/no-rec & 0.362 & 0.937 & 0.857 & 0.791 & 0.756 & 310 \\
Instantaneous/no-rec & 0.355 & 0.929 & 0.831 & 0.750 & 0.676 & 607 \\
\hline
\end{tabular}

Table 5: Results as in Tab. 2, but only events surviving a thrust cut $0.8<T<0.9$. Energy is $172 \mathrm{GeV}$; no ISR.

\begin{tabular}{|l|c|c|c|c|}
\hline & \multicolumn{4}{|c|}{ particle species and $p$ range $(\mathrm{GeV})$} \\
\hline model & $\begin{array}{c}\text { charged } \\
\text { all }\end{array}$ & $\begin{array}{c}\text { charged } \\
0.15-0.7\end{array}$ & $\begin{array}{c}\mathrm{K}^{ \pm} \\
0.15-0.7\end{array}$ & $\begin{array}{c}\mathrm{p}, \overline{\mathrm{p}} \\
0.15-0.7\end{array}$ \\
\hline no-reconnection & 10.715 & 5.843 & 0.319 & 0.108 \\
\hline $\mathrm{I} /$ no-rec & 0.979 & 0.970 & 0.948 & 0.926 \\
$\mathrm{II} /$ no-rec & 0.986 & 0.977 & 0.953 & 0.928 \\
$\mathrm{II} /$ no-rec & 0.982 & 0.972 & 0.950 & 0.927 \\
\hline 'GH'/no-rec & 0.924 & 0.892 & 0.834 & 0.747 \\
Intermediate/no-rec & 0.791 & 0.779 & 0.709 & 0.644 \\
Instantaneous/no-rec & 0.770 & 0.752 & 0.676 & 0.578 \\
\hline
\end{tabular}

Table 6: The same observables as in Tab. 2, but only for soft particles at least $30^{\circ}$ away from a jet direction. Energy is $172 \mathrm{GeV}$; no ISR.

events, so is of no practical interest. The first column shows the number of WW events surviving this cut. The ratios have little of the hoped-for enhancement; in some models the effects are even reduced. A more detailed study of different thrust bins offer little hope: in the realistic models, it is difficult to find any pattern at all. In the intermediate and instantaneous models, the reduced effects are intertwined with a change of the thrust distribution itself. We remind that low thrust is not only a signal of well-separated W decay axes but also of energetic gluon emission or fluctuations in the fragmentation process towards higher multiplicities. Therefore it is possible for a subdivision by thrust to group events of similar multiplicity, and hence to reduce visible effects in the thrust-binned momentum spectra.

One could try to enhance the signal by measuring soft particles in the interjet valleys only. Analogously to the string effect in three-jet events of $\mathrm{e}^{+} \mathrm{e}^{-}$annihilation [29] these regions of the phase space are the ones most affected by the Lorentz-boost effects. Tab. 6 gives the results, corresponding to the additional selection requirement of particles lying at least $30^{\circ}$ away from a jet direction, at $172 \mathrm{GeV}$. Comparison between the numbers in Tabs. 2 and 6 shows a certain rise of the signal in the latter case, but it is not dramatic. To be more precise, the signal is increased about equally much, relative to the results for all charged particles, by either of the restrictions $0.15<p<0.7 \mathrm{GeV}$ and 
$\theta>30^{\circ}$. Combining the two restrictions does not give much further, presumably since higher-momentum particles in the regions between the main jets carry information about the perturbative gluon emission more than about the soft cross-talk.

In summary, neither of the strategies considered above leads to a drastic improvement of the observability of the cross-talk signal. Finding better selection procedures is still a challenging task, but at the moment we have no good proposal.

As a final exercise, we discuss below the possibility of using $\mathrm{Z}^{0}$ decays for calibration of the low-momentum spectra in the no-reconnection scenario. Recall that, so far, the experimental strategy of searching for interconnection has been based on a comparison between the charged particle distributions in the (4q) decay channel with those in twice the $(2 \mathrm{q})$ mode, e.g. [22, 23]. However, such a procedure has certain caveats. Firstly, the $(2 q)$ results have their own statistical and systematic uncertainties. Given that both event samples are of comparable size, the statistical error in a comparison of the (4q) with the $(2 q)$ channel is larger by at least a factor $\sqrt{2}$ than the error in the (4q) channel itself. Secondly, the event selection cuts could introduce a bias between the purely hadronic and the mixed WW decay modes. Therefore, it looks quite appealing to try to develop a simple model-independent procedure of replacing the measured soft-particle spectra in the individual, isolated $\mathrm{W}$ decay by spectra derived from the $\mathrm{Z}^{0}$ hadronic results.

In order to acquire an appropriate ' $Z Z$ ' reference set for comparisons with the WW events, we have applied the following procedure. First a $\mathrm{W}^{+} \mathrm{W}^{-}$event is generated, using standard matrix elements as relevant at LEP2. Both W's decay hadronically. All such events are used in the analysis, i.e. there are no cuts to single out events with four wellseparated jets. This is an attempt to stay in the spirit of a totally inclusive measurement, although experimentalists would certainly introduce some cuts to reduce background. The boost factors of the two W's and the directions of the respective q $\bar{q}$ event axis in the rest frame of its $\mathrm{W}$ is then found. These numbers (two boost magnitudes and two polar angles, plus two azimuthal angles that are irrelevant for inclusive $\mathrm{d} n / \mathrm{d} p$ spectra) are then applied to two $\mathrm{Z}^{0}$ 's, each of which is taken to have the fixed mass $M_{\mathrm{Z}}=91.2 \mathrm{GeV}{ }^{7}$ This means that, for each $\mathrm{W}^{+} \mathrm{W}^{-}$event, a matching $\mathrm{Z}^{0} \mathrm{Z}^{0}$ event is found that can be analyzed in the same way. The $\mathrm{Z}^{0}$ flavours are chosen according to the relevant mixture, including b quarks. When ISR is included, each 'ZZ' event is assumed to have the same set of ISR photons as the related WW event from which the boosts and decay angles were adopted.

In an experimental analysis, the strategy above could be used to generate a large 'ZZ' reference sample, with known boosts and decay angles, and making use of a $\mathrm{Z}^{0}$ generator (such as the PYTHIA/JETSET one here [27]) that has been extensively tuned to existing $\mathrm{Z}^{0}$ data. An alternative is to reconstruct boosts and angles from the observed (4q) events, and to each such event assign a number of matching pairs of observed $\mathrm{Z}^{0}$ events. (Given the enormous $\mathrm{Z}^{0}$ statistics and the small $\mathrm{W}^{+} \mathrm{W}^{-}$one, there is no reason to stay with a one-to-one assignment of events.)

Some results from the application of the proposed recipe for comparing the WW (noreconnection scenario) events with the 'ZZ' ones are given below. The plots in Fig. 6 are for an $172 \mathrm{GeV}$ energy, without ISR. Fig. 6a,c show $\mathrm{d} n^{\mathrm{ch}, \mathrm{K}+\mathrm{p}} / \mathrm{d} p$ distributions in the range $0<p<2 \mathrm{GeV}$ for $\mathrm{WW}$ and for ' $\mathrm{ZZ}$ '. In Fig. $6 \mathrm{~b}$,d the ratio of the two, WW/'ZZ', is displayed. The plots demonstrate that there is slightly more soft-particle multiplicity

\footnotetext{
${ }^{7} \mathrm{No}$ attempt is made to rescale the momenta in $\mathrm{Z}^{0}$ events to reduce the total energy from the $\mathrm{Z}^{0}$ to the $\mathrm{W}$ mass. Such a rescaling could be relevant for a comparison of high-momentum particles, but would be misleading in the current studies. A rescaling would imply, e.g., that the fragmentation $p_{\perp}$ would be smaller in the rescaled $\mathrm{Z}^{0}$ events than in proper $\mathrm{W}$ events, contrary to the universality normally assumed.
} 

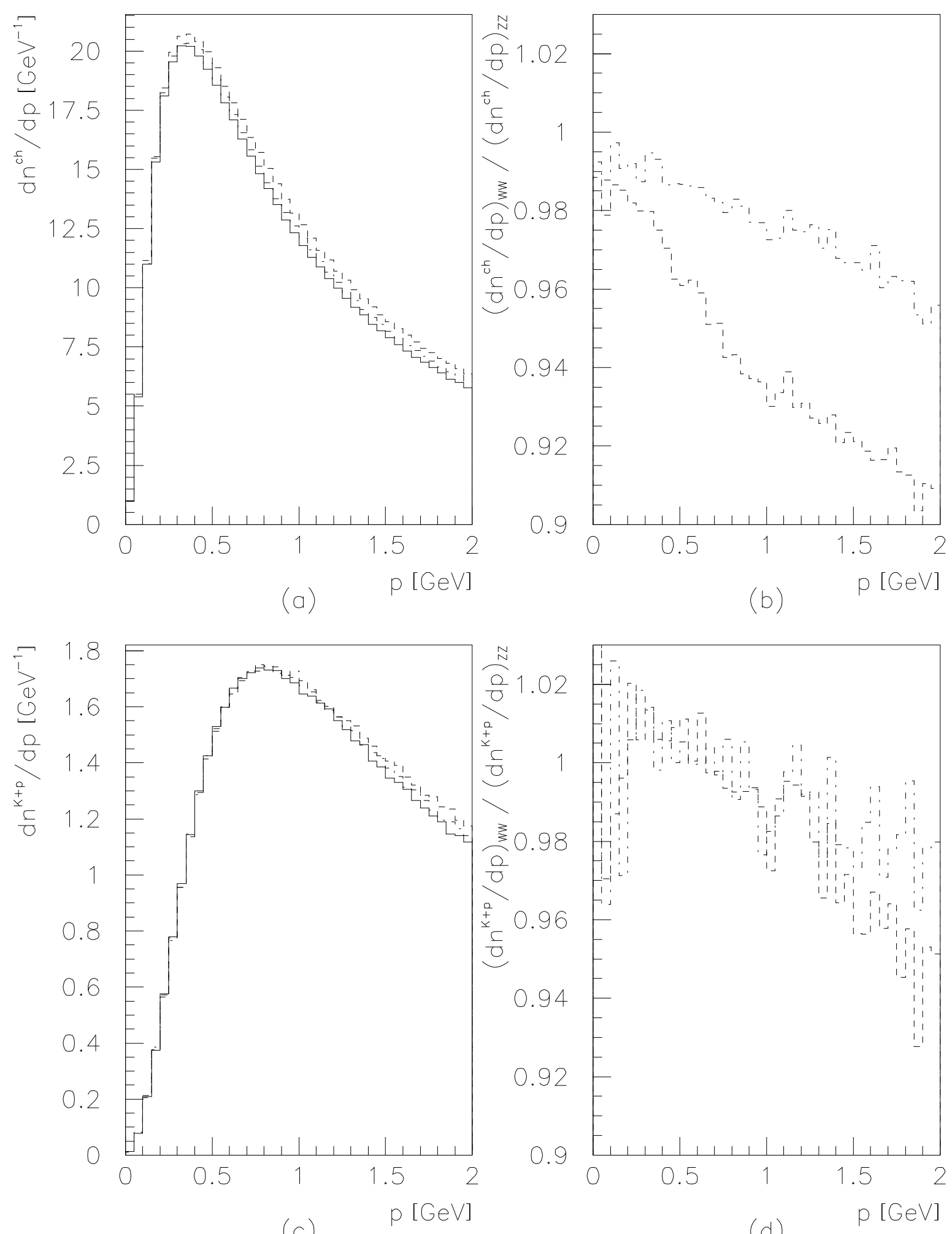

Figure 6: A comparison of momentum spectra in WW, 'ZZ' and ' $Z_{\text {nb }}$ ' events. a) $\mathrm{d} n^{\mathrm{ch}} / \mathrm{d} p$ spectra for the three models: WW full, ' $\mathrm{ZZ}$ ' dashed and ' $\mathrm{ZZ}_{\mathrm{nb}}$ ' dashed-dotted. b) Ratios of these spectra: $\mathrm{WW} / \cdot \mathrm{ZZ}$ ' dashed and $\mathrm{WW} / \mathrm{ZZ}_{\mathrm{nb}}$ ' dashed-dotted. c) $\mathrm{d} n^{\mathrm{K}+\mathrm{p} / \mathrm{d} p}$ spectra for the three models: notation as in $a$ ). d) Ratios of these spectra: notation as in $c$ ). All results are for $172 \mathrm{GeV}$; no ISR. 
in the 'ZZ' than in the WW events in the $p<1 \mathrm{GeV}$ region (an excess of $\sim 4 \%$ for all charged particles, but no net effect for kaons and protons). This is caused by the larger QCD evolution scale ( $\mathrm{Z}^{0}$ mass as compared to the $\mathrm{W}$ mass) and the presence of the b-quark contribution in the former case. An examination of the full momentum range shows that the $\mathrm{WW} /$ 'ZZ' ratio always remains below unity.

In order to understand the rôle of the primary quark flavour composition, we also show in Fig. 6 results where $Z^{0}$ decays to $b \bar{b}$ are excluded (' $Z Z_{n b}$ ' events). The difference is quite marked. For charged particles with $p<1 \mathrm{GeV}$ the $\mathrm{WW} / \mathrm{ZZ}_{\mathrm{nb}}$ ' ratio is only $\sim 1 \%$ below unity. Recall that such a weak dependence of low-momentum spectra on the parton cascading scale is what one should expect on the basis of colour coherence in QCD branching processes, see e.g. [25] and references therein. For the $\mathrm{K}+\mathrm{p}$ case the $\mathrm{WW} / \mathrm{ZZ}_{\mathrm{nb}}$ ' ratio becomes rather flat in a wide momentum interval, $p<10 \mathrm{GeV}$. For $p<1 \mathrm{GeV}$ this ratio is again almost unity.

To test the possible energy dependence we plot in Fig. 7 the same observables as in Fig. 6, but at $\sqrt{s}=195 \mathrm{GeV}$. A comparison of these plots shows that, at low momenta, the $\mathrm{WW} /$ ' $\mathrm{ZZ}$ ratio is almost energy-independent, but slightly closer to unity for higher energies. Studies in the energy range $\sqrt{s}=160-195 \mathrm{GeV}$ demonstrate that, for $p<1 \mathrm{GeV}$, the ratio rises with $\sqrt{s}$ by at most $1 \%$.

To quantify further the applications of the proposed recipe for the calibration of softparticle spectra we give in Tabs. 7 and 8 the numbers corresponding to different requirements, at 172 and $195 \mathrm{GeV}$, respectively, with ISR included. In fact, as already noted above for the other studies, the inclusion or not of ISR has a negligible impact on any of the ratios between models. Notice that the b-quark contribution increases the multiplicity in the 'ZZ' sample, but the WW/'ZZ' ratio for soft kaons remains quite close to unity (within $\sim 1 \%$ accuracy) for both the ' $\mathrm{ZZ}$ ' and ' $\mathrm{ZZ}_{\mathrm{nb}}$ ' options. As for the study of reconnection, thrust cuts do not have any significant impact on the WW/'ZZ' comparison.

The message from examining the results, presented in Figs. 6 and 7 and in Tabs. 7 and 8 , is that the proposed procedure for calibration of the WW no-reconnection sample should allow a reference for the low-momentum spectra within an accuracy of $\sim 3 \%$ for charged particles and $\sim 1 \%$ for $\mathrm{K}+\mathrm{p}$. It is also worthwhile to mention that the $\mathrm{WW} /{ }^{\prime} \mathrm{ZZ}$ ' ratio is almost always slightly below unity.

Bearing in mind that, within the realistic scenarios, the expected depletion of lowmomentum spectra as compared to the no-reconnection case is $\sim 2 \%$ for charged particles and $\sim 5 \%$ for $\mathrm{K}+\mathrm{p}$, the advocated calibration recipe, at first sight, does not look safe. However, we would like to recall that the essential part of the deviation of the WW/' $\mathrm{ZZ}$ ' from unity is caused by the b-quark effect. The latter is quite well controllable within the existing Monte Carlo models, so it looks like a straightforward procedure to correct for it. Conceivably one would even dare trust generators to have (most of) the remaining differences between WW and 'ZZ' under control, so that further corrections could be applied. The situation is even more favourable for $\mathrm{K}+\mathrm{p}$, where the reconnection-induced shifts are expected to be larger and the deviation of the ratio from unity is smaller. So there could be some prospects of a reliable application of such a normalization recipe, but further work (in particular, on the experimental side) is needed. 

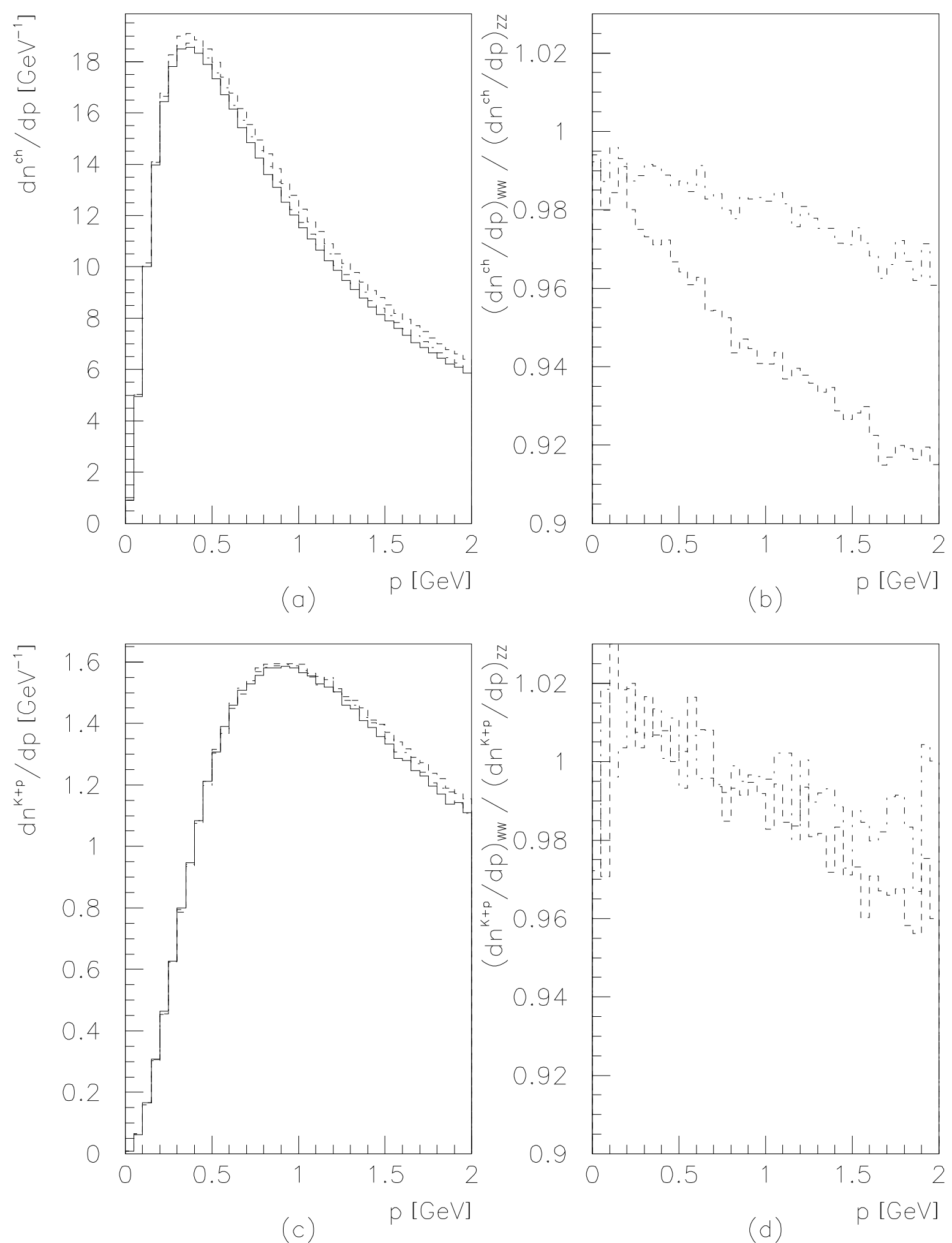

Figure 7: A comparison of momentum spectra in WW, 'ZZ' and ' $Z_{\text {nb' }}$ ' events, as in Fig. 6 but at an energy of $195 \mathrm{GeV}$. No ISR. 


\begin{tabular}{|c|c|c|c|c|}
\hline & \multicolumn{4}{|c|}{ particle species and $p$ range $(\mathrm{GeV})$} \\
\hline \multirow[t]{2}{*}{ model } & charged & charged & $\mathrm{K}^{ \pm}$ & $\mathrm{p}, \overline{\mathrm{p}}$ \\
\hline & all & $0.15-0.7$ & $0.15-0.7$ & $0.15-0.7$ \\
\hline WW (no-rec) & 38.215 & 10.121 & 0.494 & 0.161 \\
\hline 'ZZ' & 41.449 & 10.450 & 0.496 & 0.157 \\
\hline WW/'ZZ' & 0.922 & 0.968 & 0.997 & 1.026 \\
\hline 'ZZZ & 40.255 & 10.245 & 0.494 & 0.159 \\
\hline $\mathrm{WW} / \mathrm{ZZZ}_{\mathrm{nb}}$ ' & 0.949 & 0.988 & 1.001 & 1.014 \\
\hline
\end{tabular}

Table 7: A comparison of soft-particle production between WW, 'ZZ' and ' $Z Z_{\mathrm{nb}}$ ' events. Energy is $172 \mathrm{GeV}$; with ISR.

\begin{tabular}{|l|c|c|c|c|}
\hline & \multicolumn{4}{|c|}{ particle species and $p$ range $(\mathrm{GeV})$} \\
\hline model & $\begin{array}{c}\text { charged } \\
\text { all }\end{array}$ & $\begin{array}{c}\text { charged } \\
0.15-0.7\end{array}$ & $\begin{array}{c}\mathrm{K}^{ \pm} \\
0.15-0.7\end{array}$ & $\begin{array}{c}\mathrm{p}, \overline{\mathrm{p}} \\
0.15-0.7\end{array}$ \\
\hline $\mathrm{WW}$ (no-rec) & 38.364 & 9.435 & 0.434 & 0.128 \\
'ZZ' & 41.433 & 9.721 & 0.437 & 0.124 \\
$\mathrm{WW} / ' \mathrm{ZZ}$ ' & 0.926 & 0.971 & 0.992 & 1.032 \\
'ZZZ ' & 40.245 & 9.538 & 0.436 & 0.127 \\
$\mathrm{WW} /{ }^{\mathrm{n}} \mathrm{ZZ}$ & \\
$\mathrm{yb}$ & 0.953 & 0.989 & 0.994 & 1.006 \\
\hline
\end{tabular}

Table 8: Results as in Tab. 7, but at $195 \mathrm{GeV}$.

\section{Conclusion}

One of the main challenges facing experimental studies on the $\mathrm{W}$-mass reconstruction at LEP2 concerns systematic uncertainties caused by the strong interactions between the $\mathrm{W}^{+} \mathrm{W}^{-}$decay products. This subject has recently attracted much attention, see for reviews $[1,2,3,4,22]$.

The existing theoretical literature, based on quite different philosophies, shows a rather wide range of expectations for the shift in $M_{\mathrm{W}}$, from a few $\mathrm{MeV}$ to several hundred $\mathrm{MeV}$. The cross-talk between the W's may have an impact on various other properties of hadronic $\mathrm{W}^{+} \mathrm{W}^{-}$events as well.

Different hypotheses about the confinement dynamics may lead to different expectations for the final-state event characteristics. So, in principle, the experimental tests of hadronic interconnection between the W's - connectometry - could provide a new laboratory for probing the structure of the QCD vacuum. This important issue was first addressed in [5] and since then has become a subject of intensive discussions.

In order to establish the evidence for a cross-talk one has to find an observable which, on the one hand, proves to be quite sensitive to this effect and, on the other hand, could allow rather straightforward interpretation. The necessary requirements for such a connectometer are that the no-reconnection predictions should be well understood, and that the expected signal is strong enough to be detectable within the limited statistics of LEP2. The latter is, by no means, a simple task.

In the last year we have witnessed several experimental attempts to find an evidence of interconnection, see $[22,23]$. At the present level of statistics, no indication has been found. 
This paper attempts to quantify the expectations based on the string hadronization model [21] in terms of the distributions of low-momentum hadrons. This idea is motivated by an observation [6] that it is the soft particles that are most sensitive to hadronic cross-talk. Essential advantages of such an approach to connectometry is that here the no-reconnection case can be well described, and that there is no (direct) dependence on the jet reconstruction method or event selection strategy.

Studies are presented for six reconnection scenarios. Model I is based on a bag-like model for strings, and II and II' on a vortex-line approach. In $\mathrm{II}^{\prime}$ and 'GH' reconnections have to reduce the string length. For comparisons with the first three models, which have a $25-35 \%$ reconnection rate, the ' $\mathrm{GH}$ ' deviations from the no-reconnection reference should be scaled down by about a factor of 3. An unscaled 'GH' with (close to) unit reconnection probability is to be considered as an extreme. This is even more the case for the intermediate and instantaneous scenarios, which should be viewed as toy models, convenient for technical and reference purposes only. Let us therefore be very clear here: the last three lines of Tabs. 1-6 are so easy targets and of so small intrinsic value that no prize will be handed to experimentalists shooting them down. The real targets are represented by the I, II and $\mathrm{II}^{\prime}$ (and ' $\mathrm{GH}$ '/3) numbers.

It is of some interest to note that, for the four realistic models, the depletion of the low-momentum particle spectrum rather closely agree. Since the depletion should scale proportionately to the reconnection probability, we may here have a good gauge for this probability. But, as already emphasized, the models still disagree on the value of the $\mathrm{W}$ mass shift, so we cannot offer a recipe for turning a given level of depletion into a correction on the $\mathrm{W}$ mass.

The designated objective of LEP2 is to deliver $500 \mathrm{pb}^{-1}$ of luminosity. This means about $4000 \mathrm{WW} \rightarrow 4 \mathrm{q}$ events per experiment. Noting that the number of charged particles in the $0.15-0.7 \mathrm{GeV}$ momentum bin follows a (close to) Poissonian distribution with a mean of $\approx 10$, this would give a statistical error of $\delta n \approx \sqrt{10} / \sqrt{4000}=0.05$ and a relative error of $\delta n / n \approx 0.005$. The expected effect in realistic models is $\sim 0.02$ at $172 \mathrm{GeV}$ and $\sim 0.01$ at $195 \mathrm{GeV}$. From a purely statistical point of view we could therefore expect at most a $4 \sigma$ deviation and more likely $2 \sigma$, given the intended higher energy of future runs. It would be difficult to claim success based on such numbers. There are two points in favour, however. One is the presence of four experiments with independent statistical samples. The other is that we already believe to know the sign of the effect we are looking for.

Of course, given the marginal statistical significance of a signal, it is important to limit systematic errors. At first sight, this seems rather difficult. The obvious control sample of $(2 q)$ decays of $\mathrm{W}$ pairs suffers from as severe a statistics problem as the (4q) decays; actually a factor 2 worse since one needs two (2q) events to simulate one (4q) one. It would therefore be an advantage if instead one could use the essentially unlimited $Z^{0}$ sample as reference. We have shown that a straight comparison is not accurate enough, but that a correction for $\mathrm{Z}^{0} \rightarrow \mathrm{b} \overline{\mathrm{b}}$ decays almost brings one there. Presumably generators are good enough at modelling differences between $\mathrm{W}$ and $\mathrm{Z}$ decays that one would trust them also for some further corrections, making the $\mathrm{Z}^{0}$ calibration method the best bet and probably bringing this kind of systematic errors below the level of statistical ones. Other kinds of systematic errors are not considered in this article but may pose formidable challenges to the experimentalist. Of some small consolation is that our use of rather inclusive quantities implies a limited dependence on the details of the event selection cuts.

As an alternative to considering all charged particles, one may focus on the $\mathrm{K}+\mathrm{p}$ 
subsample in the $0.15-0.7 \mathrm{GeV}$ momentum range. The relative statistical error then is increased to $\delta n / n \approx 0.02$, and the expected signal to $\sim 0.05$ at $172 \mathrm{GeV}$ and $\sim 0.025$ at $195 \mathrm{GeV}$. From a statistical point of view this is a clear degradation, but it appears that the calibration to the $\mathrm{Z}^{0}$ reference sample can be made with higher accuracy. While maybe not a signal in its own right, $\mathrm{K}+\mathrm{p}$ thus might appear in the 'supporting cast', should a signal be found for all charged particles in the low-momentum range. Another similar example is the number of particles, at all momenta, that are not within $30^{\circ}$ of a jet, while cuts on the thrust of events does not seem to offer anything.

Our choice of momentum range $0.15-0.7 \mathrm{GeV}$ could be modified. The lower cut is purely a matter of convenience, since so few particles have $p<0.15 \mathrm{GeV}$ anyway. The upper cut could be increased to $1 \mathrm{GeV}$, say, giving a decrease in the average level of the signal but an increase in the number of particles contributing to it. The net effect is a slight increase in the purely statistical significance, within the context of the reconnection models studied, but probably at the expense of a somewhat worsened systematic uncertainty. A restriction to $p<0.4 \mathrm{GeV}$, on the other hand, reduces the statistics so much that the increase in the average level of the signal could not really compensate. Anyway, the message is that experimentalists should feel free to pick cuts based on a complete overview of all issues, experimental and theoretical, while we here have only attempted to discuss the latter ones. Studies for the $\mathrm{W}$ mass determination, in particular, may require rather special event selection cuts. More relaxed cuts could then be considered for the inclusive spectrum, but is not an absolute prerequisite.

Our studies do not encourage a too optimistic prognosis concerning the prospects of connectometry on the basis of low-momentum spectra, even having the whole aimed-for statistics of LEP2. The best we can hope is that the expected signal would be at the edge of observability. In such a case one would need a lot of hard work (and good luck) in order to detect the signal reliably. However, we would like to emphasize that, given the present lack of deep understanding of the non-perturbative QCD dynamics, it is only experiment that could lead the way and may cast light on the challenging issues of the hadronic cross-talk.

We would like to make it absolutely clear that the nonobservation of the reconnection effects on the low-momentum spectra, by no means, indicates their nonexistence. Most likely it may just mean that the "queen of observables" is still to be nominated.

It may be useful here to recall some other ideas. We are not saying that either of them are offering larger hope than the currently studied one, but they could add pieces to the jigsaw puzzle:

- In the string model, particles are produced at limited transverse momenta with respect to the string direction (in the rest frame of the string). One may therefore attempt an event-by-event reconstruction of the colour topology by a minimization of the total transverse momentum with respect to the potential sets of strings [31]. The inclusion of parton-shower activity smears the signal significantly, but still effects are at a comparable level of statistical significance to the ones reported here. The chances of controlling systematics appear to be worse, however. Also in this approach most of the information content is sitting in low-momentum particles, roughly out to $3 \mathrm{GeV}$.

- A more inclusive test of colour topology is the azimuthal distribution of particles around jet directions [6]. Since the two jets of a W are approximately back-to-back, to zeroth approximation one would expect a flat distribution. If a reconnection occurs, the new strings would be spanned in the direction of the two jets from the 
other W. In practice one could, for each jet, define $\varphi=0$ as the direction to the nearest other jet. The signal for reconnection would then be an enhanced particle production close to $\varphi=0$ and $\varphi=\pi$, and a reduced one at around $\varphi=\pi / 2$. However, this effect has to be extracted from a much larger non-isotropy induced by simple kinematical effects, e.g. that the jet clustering procedure depletes the particle production in the direction towards the next nearest jet. It has not been studied whether the signal could be enhanced by suitable cuts, e.g. a restriction to low-momentum particles.

- A variant of the azimuthal anisotropy study is to concentrate on activity out of the event plane of the two jets of a W. In the absence of reconnections, and neglecting hard perturbative gluons, particle production is concentrated to the event plane of each W. A reconnection would increase the activity out of the plane.

- The reduced low-momentum particle production has to be compensated by an increase elsewhere, in order to conserve energy. We have above noted that, in the realistic models, most of the effect occurs at intermediate momenta, say $1.5-5 \mathrm{GeV}$. At higher momenta both the original and the reconnected strings line up particles with the jet directions and have little memory of the central overlap region. The compensation effects are rather small, since fewer particles need be added at intermediate momenta to give the same amount of energy as the one taken away at low momenta. Given that experimentalists also measure in this range, there is a possibility to increase the potential significance of a signal. The calibration to a no-reconnection scenario, e.g. based on $\mathrm{Z}^{0} \mathrm{Z}^{0}$ events, would be even more delicate in this region, however.

It is important to bear in mind, that even if/when the evidence for the reconnection signal is established, it may be quite a long way before one would be able to draw a convincing conclusion concerning the W-mass shift.

Finally, let us recall that the reconnection probability is a free parameter in the majority of the models. So if forthcoming results from LEP2 show shifts which are significantly greater than our expectations, this could signal that the reconnection probability is larger than in the models adopted in this paper.

\section{Acknowledgments}

We would like to thank A. De Angelis, M. Battaglia, A. Blondel, D. Liko, N. Neufeld, R. Orava, B. Pietrzyk, A. Tomaradze, N. Watson and B.R. Webber for useful discussions. VAK thanks the Theory Division of CERN for the warm hospitality during the course of this work. This work was supported in part by the EU Fourth Framework Programme 'Training and Mobility of Researchers', Network 'Quantum Chromodynamics and the Deep Structure of Elementary Particles', contract FMRX-CT98-0194 (DG 12 - MIHT). 


\section{References}

[1] W mass working group, Z. Kunszt et al., in "Physics at LEP2", eds. G. Altarelli, T. Sjöstrand and F. Zwirner, CERN Report 96-01 (1996), Vol. 1, p. 141.

[2] QCD event generators working group, I.G. Knowles et al., ibid., Vol. 2, p. 103.

[3] B.R. Webber, talk at Phenomenology Workshop on LEP2 Physics, Oxford, April 1997, hep-ph/9708463.

[4] A. Ballestrero et al., "Report of the working group on W mass and QCD", hep$\mathrm{ph} / 9709283$.

[5] G. Gustafson, U. Pettersson and P. Zerwas, Phys. Lett. B209 (1988) 90.

[6] T. Sjöstrand and V.A. Khoze, Z. Phys. C62 (1994) 281; Phys. Rev. Lett. 72 (1994) 28.

[7] G.Gustafson and J.Häkkinen, Z. Phys. C64 (1994) 659.

[8] L. Lönnblad, Z. Phys. C70 (1996) 107.

[9] B.R. Webber, in [2], p.161.

[10] Š. Todorova-Nová, DELPHI Internal Note 96-158 PHYS 651.

[11] J. Ellis and K. Geiger, Phys. Rev. D54 (1996) 1967; Phys. Lett. B404 (1997) 230.

[12] J. Häkkinen and M. Ringnér, Lund preprint LU TP 97-32, hep-ph/9711212.

[13] L. Lönnblad and T. Sjöstrand, Phys. Lett. B351 (1995) 293.

[14] S. Jadach and K. Zalewski, Acta Phys. Polon. B28 (1997) 1363;

V. Kartvelishvili, R. Kvatadze and R. Møller, Phys. Lett. B408 (1997) 331;

K. Fiałkowski and R. Wit, Acta Phys. Polon. B28 (1997) 2039; preprint TPJU-3-97, hep-ph/9703227;

Š. Todorova-Nová and J. Rameš, hep-ph/9710280.

[15] L. Lönnblad and T. Sjöstrand, Eur. Phys. J. C2 (1998) 165

[16] V.A. Khoze and W.J. Stirling, Phys. Lett. B356 (1995) 373.

[17] V.A. Khoze and T. Sjöstrand, Z. Phys. C70 (1996) 625.

[18] W. Beenakker, A.P. Chapovskii and F.A. Berends, Nucl. Phys. B508 (1997) 17, Phys. Lett. B411 (1997) 203.

[19] V.S. Fadin,V.A. Khoze and A.D. Martin, Phys. Lett. B320 (1994) 141; Phys. Rev. D49 (1994) 2247.

[20] L. Taylor, to appear in the proceedings of the XVII ${ }^{\text {th }}$ Int. Conf. on Physics in Collision, 1997, ed. H. Heath (World Scientific, Singapore), hep-ex/9712016.

[21] B. Andersson, G. Gustafson, G. Ingelman and T. Sjöstrand, Phys. Rep. 97 (1983) 31. 
[22] A. De Angelis, Talk at XXVIII Intern. Symp. on Multiparticle Dynamics, Frascati, September 1997.

[23] OPAL Collaboration, K. Ackerstaff et al., Eur. Phys. J. C1 (1998) 395;

DELPHI Collaboration, P. Abreu et al., DELPHI 98-16 CONF 117.

[24] for reviews see: Yu.L. Dokshitzer, V.A. Khoze, A.H. Mueller and S.I. Troyan, Rev. Mod. Phys. 60 (1988) 373; "Basics of Perturbative QCD", ed. J. Tran Thanh Van (Editions Frontières, Gif-sur-Yvette, 1991).

[25] Ya.I. Azimov, Yu.L. Dokshitzer, V.A. Khoze and S.I. Troyan, Z. Phys. C27 (1985) 65, C31 (1986) 213;

V.A. Khoze, S. Lupia and W. Ochs, Phys. Lett. B394 (1997) 179; MPI preprint MPI-PhT/97-68 (1997), hep-ph/9711392.

[26] DELPHI Collaboration, P.Abreu et al., Phys. Lett. B347 (1995) 447.

[27] T. Sjöstrand, Comput. Phys. Commun. 82 (1994) 74.

[28] L. Lönnblad, Comput. Phys. Commun. 71 (1992) 15.

[29] B. Andersson, G. Gustafson and T. Sjöstrand, Phys. Lett. B94 (1980) 211.

[30] M. Battaglia, R. Orava, D. Liko and N. Neufeld, DELPHI 98-23 CONF 122.

[31] E. Norrbin and T. Sjöstrand, Phys. Rev. D55 (1997) R5 